\title{
Using groups to change the department head role: An organization development case
}

\author{
Abstract \\ This article provides a detailed description and analysis of how one institution used groups of \\ Department Heads as change agents to address their most acute Department Head role tensions. \\ It is demonstrated that this institution's change initiative aligned very closely to the \\ recommendations proposed in both literature pertaining to organizational development in a \\ higher education context and the broader organization development literature regarding the use \\ of groups as agents of change. In conclusion it is suggested: that how work is broken down and \\ structured into various groups can facilitate the change process, that enabling and engaging the \\ change targets as change agents may be of value and that institutions may benefit by broadly \\ increasing member knowledge, competencies and experience with team work, team dynamics \\ and an understanding of how groups can successfully be used as agents of change.
}

\section{Keywords}

Organization development, organizational change, change management, higher education, department head

\section{Introduction}

Academic institutions evolve and change over time. As an institution's mandate shifts, so must its organizational structures, policies, procedures and culture if it is to reach its full potential. However organizational change in higher education is complicated. Higher education 
organizations are highly complex and differentiated (Clark, 1983), are known for their suboptimal decision-making processes (e.g. Baldridge, 1971) and have been broadly criticized for inefficiency, indifference to external constituencies, and resistance to change (Ruben, 2005). The long history and deeply imbedded but decentralized culture of many higher education institutions also make broad based organizational changes very challenging. That said, there are also cases of organizational development and turnaround that speak against resistance, and provide some evidence that higher education institutions are indeed able to change (see e.g. Sporn, 1996; Clark, 2001; Norgaard et al., 2001). But it is far from clear from the emerging literature, which factors are crucial catalysts or important process variables that support change initiatives. The literature contends that, for instance, leadership is important (Clark, 2001), but is less clear on the how and when.

In this article we present a detailed description and analysis of how Mount Royal University used groups of Department Heads as change agents to address their most acute Department Head role tensions with the intent to strengthen academic leadership behaviour. While academic research has described many of the current roles, responsibilities, tensions and identities of Department Heads (Bryman, 2007, Chu, 2006, Henkel, 2000, Lees, 2006, Seagren et al., 1993) it has not attempted to clarify ways for institutions to address key issues related to the position (London, 2011), one exception being a recent article by Berdrow (2010).

Our account aims to provide a detailed description and analysis of what Mount Royal did to address the key tensions associated with their Department Head role and to reflect on how the 
processes and structures utilized impacted their change effort. While not consciously done by the Mount Royal team, we demonstrate that this institution's change initiative aligned very closely to the recommendations proposed in both literature pertaining to organizational development (OD) in a higher education context and the broader OD literature regarding the use of groups as agents of change. Moreover, how the transformation of the Department Head role took place at Mount Royal provides an explicit example of how these collective findings and recommendations from the literature translated into practice at a higher education institution.

The structure of the article is as follows. First an overview of OD literature in a higher education context and literature pertaining to using groups as agents of change is discussed. Then a detailed case study and associated reflections are presented. Finally, a number of implications for organizational development initiatives in a higher education context are offered.

\section{Organizational Development in Higher Education}

Organization development (OD) has traditionally been defined as the use of behavioral science methods to achieve system-wide change (Beckhard, 1969, Beer, 1980, Cummings, 2008). While the body of literature available in the broader field of OD has been growing, there are very few OD case studies in a higher education context (Kezar et al., 2011, Kezar and Eckel, 2002).

Torraco and Hoover (2005) summarize the literature regarding the use of OD approaches to change in universities based on seven university cases. From this work, they offer a number of 
lessons learned and implications for administrators, change leaders and change agents. These include the importance of: a needs assessment before starting the initiative, support from senior leadership, strong change leadership, monitoring tensions between administration and faculty, identifying highly motivated and persistent change supporters and getting them involved, but also the importance of involving everyone in the organization in the process in some way and designing or adapting a process that fits the organization's culture (Torraco and Hoover, 2005).

Based on case studies of five UK institutions transitioning from colleges to universities, McRoy and Gibbs (2009) advocate a form of "contextualized leadership" that is relevant to the institution under change. They argue that the leadership and management practices must fit with the contextual environment to enable change. They also emphasize the importance of senior leadership support. They found that change agents were mainly concerned that senior management was fully supportive of their role (McRoy and Gibbs, 2009).

Based on his experience in the Center for Organizational Development and Leadership at Rutgers University, Ruben (2005) offers a number of lessons learned and general principles for implementing organizational development programs in higher education institutions. While somewhat incorporated into the findings provided by Torraco and Hoover (2005), Ruben (2005, pp.383-388) provides a number of guidelines for consideration. Ruben (2005) first advises to be extremely cautious in adopting programs from other institutions and sectors. He warns many programs go by the wayside because they adopted, rather than carefully adapted, programs from other places and/or times (Ruben, 2005). He further elaborates that there is danger promoting an initiative as "new, unique or special", as it can trigger defensiveness and resistance from various 
individuals and departments. His recommendation is to "build on, link to, and leverage the successful work of others" (Ruben, 2005, p.383).

The language, terms and titles used to describe an initiative "may be one of the most important decisions to be made" (Ruben, 2005, p.385). Customary language of the corporate world is potentially problematic. Terms such as customer focus, reengineering, benchmarking, and performance measurement may disengage many members of the institution. "The difference between the sound and resulting impact of customer focus and student focus can be dramatic, although from an organizational development perspective, the implications of the two are essentially the same" (Ruben, 2005, p.386) Ruben argues, much can be gained and little lost by using the terms' more familiar counterpart in the customary language of higher education.

\section{Implementing Change with Groups}

"Groups are prominent in the field of organizational development" (Hackman and Edmondson, 2008, pg. 167) and are used as tools for organizational change. From the broader OD literature, Hackman and Edmondson (2008) provide insight regarding the use of groups as agents of change and what can be done to increase their chances of success. They describe the central challenge of designing and leading change teams as tied to the requirement of the group to do two things at once: get specific change tasks accomplished and continuously learn and adapt in a context that is inherently fluid and unpredictable. 
Based on a consolidation of findings from their research, Hackman and Edmondson (2008) describe five main conditions that, if in place, should support both the task performance required and continuous learning required by group-led change initiatives. First, the group must have a clear and challenging vision; one that is compelling and meaningful but also requires significant effort and uses the members' knowledge, skill and experience. Second, the group requires appropriate boundaries. The ideal is a group that has distinct but permeable boundaries, which permit both continuity to pursue change goals and cross-boundary activity that can produce appropriate change strategies and tactics and self-correct when plans go off track. Third, the group requires sufficient autonomy to manage both their own processes and their relationships with external individuals and groups, but not so much that members feel the group can deviate substantially from agreed-upon change objectives or from organization norms and values. Fourth, the group requires an organization culture that supports change as organizational diagnosis and change activities require interaction with and support from the broader organization. To enable a change team, Hackman and Edmondson (2008) propose the group should clearly be an independent entity with a membership which roughly mirrors the makeup of the larger targeted group. Finally, the group requires change leadership that fosters both performance and learning. Leaders of change groups are challenged with creating a safe internal climate that supports a focus on task requirements and performance, while maintaining a shared commitment to explore and learn from new ideas, dissenting views, and unanticipated problems and opportunities (Hackman and Edmondson, 2008, pg.178-183).

\section{Method}


The case study details presented throughout the remainder of this article are drawn primarily from a research project conducted by the first author, being an external to the case organization. The first author had no involvement with the consulting firm used in the beginning of the development project or with the development project itself. The initial research project included two semi-structured interviews with both the project sponsor (the V.P. Academic) and the initiative team lead (an Associate Dean) and single semi-structured interviews with two taskforce members (Department Heads). The interviews were conducted between May and August 2011. The interview guides were designed on the basis of the analysis of the literature to capture details about the change initiative and compare and contrast it to the lessons learned and recommendations from the organizational development literature. A detailed review of a wide range of official working documentation (such as committee charters, meeting minutes, taskforce reports and pamphlets) was also completed between June and October 2011.

The second author, who was the change initiative team lead, provided additional detail and clarification to assist in converting the completed research project to a journal article. No fundamental additions or changes were made to the original work.

There are limitations of case studies. For example, "case studies depend on our willingness to accept the personal perspective of the author and its impact on data gathering, the provisional nature of truth, and the imperfections of historical reconstruction" (Barker, 2006, p. 280). However to increase construct validity, the interviewees were consciously chosen and background documentation carefully selected in an attempt to triangulate data from multiple 
sources and multiple perspectives. The two Department Heads were also chosen to represent varying viewpoints. Prior to the change, one was identified as a potential skeptic of the change initiative and the other was identified as a likely supporter of the change initiative. To increase the likelihood that interviewees would be comfortable to provide truthful responses, all were offered anonymity. Also, responses from the interviewees were compared and contrasted to determine reliability of the findings.

\section{The case of Mount Royal University}

Mount Royal University, located in Alberta, Canada, has seen its mandate evolve noticeably, from its start over 100 years as a faith-based college, to a community college, to a university transfer college and, most recently, to a degree-granting university. Mount Royal currently has over 13,500 full-time students enrolled in 61 credit academic programs that are organized within 7 Faculties. There are 30 academic departments comprised of a total of 404 full-time instructors and 542 part-time instructors. The institution is supported by over 700 staff and 89 individuals within management positions. Academic departments range in size from a department of 50 fulltime faculty members to two departments of less than 10 full-time faculty. The head of the academic affairs of the institution is the Vice-President Academic, with Deans responsible for the 7 Faculties and 30 Department Heads reporting to the Deans.

Government legislation changed Mount Royal University from a college to a primarily regional institution that provides baccalaureate degrees with a focus on teaching and applied research in 2006. In 2010 it was officially renamed by the state accrediting body as a university. In 
anticipation of the most recent mandate shift, a number of organization development initiatives were sponsored by the V.P. Academic [the Chief Academic Officer or Pro-Vice-Chancellor equivalent]. These initiatives included the creation of new governance structures with higher levels of faculty involvement and various activities focused on evolving the roles of faculty to embrace a research function.

\section{Department Head initiative overview}

In early 2007 Mount Royal University established a change initiative centered on the Department Head role. They wanted their Department Heads "to focus on different things" (V.P. Academic,

pers. comm., $27^{\text {th }}$ Jun., 2011). The activities undertaken and the changes made addressed a number of the key tensions such as the lack of clarity regarding the Department Head's authority and poorly planned transitions as new Department Heads assumed the role. With these tensions relieved, although not completely eradicated, the parallel activities which would develop the competencies and provide support for the desired increased academic leadership behaviours were able to be implemented.

During the last four years, four groups have been chartered to work on this initiative. These groups emerged over time as the leadership and coordination needs of the change initiative evolved. The first group had a mandate to develop a 3-year work plan to address the most consequential issues for Department Heads; a "diagnostic group" using Hackman and Edmondsons' (2008) term. The second was an implementation team tasked with implementing the recommendations from the first group. The final two are new ongoing committee structures 
intended to sustain the change: a Department Heads Assembly and a Department Head Professional Development Committee. Each of these groups was chartered with a documented mandate, goals and representative membership criteria. Each of the groups consisted primarily of Department Heads.

\section{Recognizing the need for change}

Department Heads play a key role in the fulfillment of an institution's mission, vision and mandate. They have a "central role ...[a] pivotal function in the organization" (Spiller, 2010, p.697). Department Heads provide the link between those who are responsible for providing the core function of the institution with those who are responsible for leadership and administration of the institution (see also Floyd and Wooldridge, 1996; Meek et al., 2010). At Mount Royal, the V.P. Academic believed, "a different type [of Head] was going to be needed" as the institution shifted from a college to a university.

To better understand the issues the V.P. Academic initially hired a well-known consulting firm with significant experience in higher education to research external practices regarding the Department Head role and to perform an internal needs assessment. This included interviewing a variety of Department Heads and Deans regarding the current state at Mount Royal University and to provide a report with recommendations. The study findings identified a number of tensions such as the relatively short three-year term of Department Heads which created barriers for Department Heads to perform the desired academic leadership behaviours and it made a 
number of change recommendations. Moreover, senior administration believed "that Department Heads were spending too much time acting as paper pushers instead of academic leaders".

\section{Diagnosing the change need}

Following the consultant's report, an internal taskforce was formed and chartered with a mandate to develop a 3-year work plan to address the most consequential issues for Department Heads. One of the key pieces of guidance from the literature describing OD initiatives in higher education is the importance of identifying who is supportive of the desired change and getting them involved in the change (Cohen et al., 2005, McLean, 2005, Ruben, 2005). In this case, an Associate Dean, who recently held a Department Head role at Mount Royal and had an ongoing professional interest in the Department Head role, approached the V.P. Academic and requested the opportunity to champion the initiative. The remaining taskforce membership was a representative sample of the change targets - a group of Department Heads. It was comprised of 6 Heads, one from every Faculty or School across the institution.

The taskforce chair conscientiously sought out both supporters and skeptics when forming the group (Team Lead, pers. comm., 7 Aug. 2011). Two Department Heads that were potentially skeptics of the initiative were approached and agreed to participate. When asked to reflect upon the work of this taskforce, all interviewees commented on the importance of the team composition and how this positively contributed to the team performing their work. This is also supported by the literature as a critical component when creating groups as agents of change: "Forming inflexibly diverse groups [including representatives from key stakeholder groups and those 
with varying viewpoints] to ensure that all relevant perspectives are brought to bear on a particular change problem" is the guidance (Tucker et al., 2007, cited by Hackman and Edmondson 2008, p. 168).

Another theme that emerged from the meeting minutes, the interviews and the participating authors' experience was the noticeable support from the V.P. Academic. Not only did he speak positively and frequently about the work, he also provided physical resources and funding. This again is consistent with the guidance provided from the literature, which states, "senior administration, at least two positions above the unit administration must be in agreement and fully support the desired OD changes" (Torraco and Hoover, 2005, p.435).

Interviewees described the diagnostic group work as collaborative, however by the end of the first few meetings the group decided that they needed to deviate from their original task. The group believed that they needed to create a new Department Head job description before additional work could be started. "We didn't really know what behaviours we were looking for yet." (Group member, pers. comm. 29 $9^{\text {th }}$ Jun 2011). The group leader quickly spoke with the V.P. Academic to gain support for this deviation and, once approved, work on the job description began. This behaviour evidenced the groups understanding of their boundaries (Torraco and Hoover, 2005) but further demonstrated that the group's leader was creating a safe team environment that was able to adapt from their recommended mandate to include new information that the group felt was pertinent. 
Within a month, the taskforce had created a draft job description for the Department Head role. Each member of the taskforce was responsible for discussing the proposed description in their faculty and seeking input. The proposed description was also presented by the V.P. Academic at both the Deans and Department Heads meeting and the Executive Leadership meeting. With minor changes, the proposed Department Head job description was given approval by the Department Heads in every faculty as well as by the Deans shortly thereafter. This consultation and leveraging of the members home unit relationships to gather and vet feedback is a good example of why the composition and membership selection for the OD initiative is critical. The group members were required to act as a communication link to and from their home Faculties. They needed to engage and represent a broader constituency.

The taskforce then turned to analyzing other key tensions associated with the Department Head role. The consultant's report was reviewed, but not followed exactly. While it was a good starting point, the group ended up focusing on a few different issues that were based on input from the group and their faculty colleagues. Each member of the committee was tasked with gathering additional input and discussing the working suggestions with their respective Faculties. This approach is in alignment with the caution provided by Ruben (2005) regarding adopting programs and plans from other institutions and sectors. The group was informed by the consultant's report but not constrained by it. 
After numerous meetings, several each month, and prioritization and clustering activities, consensus on the recommendations was reached. The diagnostic team submitted a 71 page final report which articulates seven recommendations as follows:

1. Create a new role description for Heads - setting clear expectations regarding academic leadership behaviours.

2. Adjust the length of Head term from 3 to 5 years and incentivize Heads to stay in the role for the five year term with a sabbatical following completion and increased stipend in years 4 and 5.

3. Adjust recruitment and preparation of Heads

i. Appoint future Heads 6 to 12 months in advance.

ii. Provide time to the incoming Head to job shadow one term prior to occupying the role.

4. Create a performance planning and evaluation process for Heads.

5. Increase Head professional development.

6. Establish Heads meetings.

7. Revise the system of Head release time to align with current demands of the role.

The group's final report was reviewed by the V.P. Academic. He then presented and discussed the recommendations with both the Deans Council and the Executive Leadership team. The V.P. Academic- consulted broadly and sought support from interlinking constituencies. Continuous communication was an ongoing theme shared by all the interviewees regarding the- initiative, the consistent consultation and information sharing at multiple levels. The members of the group 
consulted with their respective faculty colleagues and the V.P. Academic consulted widely across the various Dean and Executive teams.

The V.P. Academic then added the implementation of changes to the Department Head role based on the groups' recommendations to the institution's Academic Plan [a strategic planning document]. This was seen as an important and symbolic form of support. It indicated to all members of the organization the priority and importance of the initiative (Group member, per. comm., 23rd Jun. 2011). From the authors' perspective, it was also equally important that this act tied the work of the implementation group to a formal reporting and oversight structure. It was an act that created the space for team work autonomy while maintaining visibility and oversight. The diagnostic group had then completed their task and the taskforce was disbanded.

While the recommendations focused on a number of concrete changes for Department Heads, the extensive conversations and consultations that occurred during the development of the report allowed for discussions of fundamental aspects of the Department Head role as well. For example, there was extensive discussion regarding the role of Department Heads as faculty members within administrative responsibilities, and whether retaining some teaching responsibilities while serving as Department Head would cause teaching and/or administration to suffer. There was considerable discourse on the efficacy of making such extensive changes to the role within a relatively short time frame. However, support for the many changes began to develop within the context of the university which was undergoing significant change at the time as it shifted its mandate from college to university status. It was recognized that for the university to become successful in its new mandate, a new level of academic leadership would be 
required from its Department Heads. Consultations provided a forum for Department Heads (and other stakeholders) to develop a shared understanding not only of the desired changes to the role, but also the rationale for evolving the role, thereby developing cultural acceptance of the revised role. Cultural transitions take time and with autonomous knowledge workers (with no strict compliance monitoring systems), behavior changes happen through buy-in. This process provided a forum for developing and internalizing new norms, values and expected behaviours associated with the Head role. Two primary outcomes of the process were: 1) implementation of the "straight-forward proposals"; and 2) the associated buy-in and cultural shift associated with changing the behaviours of those in the Department Head role.

\section{Implementing the changes}

Next the "Role of the Department Head Implementation Team" was chartered. This group was tasked with implementing the changes recommended by the initial diagnostic team. Again, this team was sponsored and provided resources by the V.P. Academic's office but, following the initial meeting guidance by the V.P. Academic, was left to do their work (Team lead, pers. comm.., $11^{\text {th }}$ Jun.2011). The team reported on their progress on a regular basis to the Deans, Department Heads and V.P. Academic and often took draft initiative outlines to their colleagues for review and feedback. Since many of the initiatives required adjustments to institutional policy and procedure, a variety of groups were involved in implementing those initiatives including Human Resources, the faculty union and senior administration. Since the initiative was included as a strategic priority within the academic plan, the team reported the status of its work regularly to the Academic Plan Implementation Committee. 
Implementation team membership. This task team's membership also consisted of a Department Head from each of the institution's Faculties but was further expanded to include three additional members: a Dean, a Human Resources Specialist and a Department Head's Administrative Assistant. This expanded group membership acknowledged the interconnectivity of the Department Head role with other institutional roles and more appropriately represented the constituents targeted for change. The literature on change speaks clearly about the importance of team membership. If team members are deliberately selected to represent key constituents targeted for change, the initiative is likely to have greater credibility, a critical factor in most higher education cultures. It further fosters legitimacy and fairness by engaging people (Beer, 1980, Kotter and Cohen, 2002). The documents indicate, five of the original six members of the diagnostic taskforce chose to participate as members of the implementation team and the Associate Dean was once again appointed the team lead.

General team process. The implementation team operated quite differently than the initial diagnostic taskforce. The group met almost monthly for approximately 2 years. The group work process consisted mainly of individual team members preparing a "working" paper on a particular issue or item and then presenting it at the group meeting for discussion. These issue owners were primarily self-selected based on personal interest or expertise. Some minor social loafing challenges were described by interviewees, however most members were diligent and timely in preparing their working papers. Discussions were described as open and respectful.

Discussing more fundamental matters (1st reviewer question) fits here I think? Perhaps discussion around the purpose bigger picture role for the chairs, how it fit into the "new 
university" world? Did it provide a forum for the creation of common understanding of where they fit into going forward? Perhaps this links to the reviewers' next comment about taking years to come out with rather straight-forward proposals. ...the story I make up in my head circles around this process being linked to the cultural transition from a college to a university. Cultural transitions take time and with autonomous knowledge workers (with no strict compliance monitoring systems), behavior changes happen through buy-in. This process provided a forum for developing and internalizing new norms, values and expected behaviours associated with the Head role. Does that makes sense or resonate based on your experience in the process? I see two outcomes of the process, one being the implementation of the "straightforward proposals" the other being the associated buy-in and cultural shift associated with changing the behaviours of those in the Head role.

For a few more complex or larger issues sub-groups were created and they reported back to the larger group. Some working paper recommendations and sub-group recommendations were quickly accepted and others required a significant amount of discussion and dialogue to reach a decision. While consensus was actively sought on all issues, it was not always achieved. When consensus could not be achieved, a voting, majority rules approach was taken. However, this was not required often. Once a course of action was decided by the group, individual members or small sub-groups were assigned the action items associated with implementing a specific recommendation. If guidance was needed or an irresolvable issue was identified, the team lead engaged the V.P. Academic for assistance on behalf of the group. 
Work produced: recommendations implemented. Implementation of the recommendations has taken a lot of time and two of the recommendations, numbers four and seven are not yet complete at the time of writing. The first recommendation, creating a new role description for Department Heads, was almost completed by the initial diagnostic taskforce. The implementation team did follow up to review institutional policies and provided some modest updates to ensure the policies were current, relevant and reflective of the Department Head job description changes.

The second recommendation, increasing the length of the Department Head term from 3 years to 5 years, had agreement in principle very quickly; however the actual implementation required renegotiating a change in institutional policy. While both faculty and administration supported the change, the bureaucracy of such a modification, outside of the standard collective bargaining cycle, required a number of meetings and a number of months to complete.

Recommendation three, the recruitment and preparation of Department Heads, was "a little easier to implement" (Group member pers. comm., 29 ${ }^{\text {th }}$ Jun 2011) as the activities had little impact outside of the academic affairs unit. One institutional policy, "Department Head Selection" did need to be updated but recommended changes were socialized by taskforce members then accepted without resistance. Release time for the incoming Department Head to job shadow the outgoing Department Head during their final term has started. There is, however, an inconsistent cycle as to when new Department Heads are appointed; therefore some academic departments have experienced the impact of this change more quickly than others. There has 
been very positive feedback on the value of job shadowing and on the Department Head Transition Checklist which has been designed to ensure certain tasks and information is exchanged between incoming and outgoing Department Heads. The literature also supports how important the transition from one leader to another is: "All those concerned with the appointment of school leaders are recommended to give more care to managing their arrival, induction and departure" (Barker, 2006, p.277).

Recommendation five, Department Head Professional Development and recommendation six, a Head's Assembly, were both brought to life via the creation of ongoing standing committees, funded from the V.P. Academic's office and lead by Department Heads. The creation of a Head's Meeting, now known as the Head's Assembly was chartered. All Department Heads are automatically members. The Speaker or chair of the Head's Assembly is elected and provided release time to lead the group. The Head's Assembly convenes a minimum of twice each term with additional meetings scheduled as needed. Although this is a new committee structure, the documentation and interviewees indicate regular attendance and participation has been the norm.

The Head's Assembly stumbled a little getting started. The V.P. Academic was initially reluctant to charter and provide resources to a committee for the Department Heads to meet independently. He was not convinced of the need. The implementation group, on the other hand, felt strongly that it would be of value to Department Heads. The V.P. Academic did concede and the committee was chartered. The interviewees shared varying perspectives on the types of issues the Head's Assembly should address however they all agreed that it has become a 
strong group that is valued by the Department Heads and "they are really getting some things done ... establishing consistency in the application of process and policies" (Group member pers. comm., 29 $9^{\text {th }}$ Jun 2011).

The Department Head professional development committee is also a chartered ongoing committee. It is responsible for providing Department Head development programming. Structurally, it reports on its activities to the Head's Assembly. The membership consists of a team leader, a representative from each Faculty and two ex officio/non-voting members, the Heads Assembly Speaker and the V.P. Academic.

Work outstanding: recommendations not yet implemented. Recommendation four, to create a performance planning and evaluation process for Department Heads, is not complete, but is expected to be ready for launch in fall 2012. Not surprisingly, creating a performance planning and evaluation process has proven to be one of the most difficult recommendations to implement. It has taken a particularly large amount of consultation to get agreement on what is needed for the process, let alone agreement on the specific tools to be used for the process. Creating an evaluation process has taken patience. It required numerous reiterations and many back and forths, with many groups. However the complexity and challenge of too many cooks in the kitchen on this sensitive task was recognized by the initial diagnostic team (Team Lead, pers. comm., 7 Aug. 2011). They had very consciously recommended that the working group for this task have no more than six members consisting of Department Heads, Deans and a Human Resources representative with hopes that a smaller but representative group would not get stuck 
in the mud. The working sub group acquired agreement on the general principles for the Department Head Evaluation Process first. They then turned their attention to creating new tools and processes needed to support implementation.

Recommendation seven, revise the formula for Department Head release time to reflect current demands of the role is not complete. The creation of new criteria and an association formula for release time has been agreed to in principle, however, the financial and resource implications of this recommendation have required a more extensive due diligence that has not been possible in all departments. As part of this due diligence the implementation team was asked by the V.P. Academic to perform a resource audit to assess the impact of implementing the changes from both a financial and human resource perspective and it was found that the current budget constraints would not allow the recommendation to be implemented institution-wide.

\section{Reflections on the Case Study}

Although this structure evolved over time at Mount Royal, upon reflection it is clear that it aligned with a number OD literature recommendations. It enabled the larger goal of increasing academic leadership behaviours of Department Heads, to be broken into more manageable pieces and by its design the work of each group built on, linked and leveraged the work of each group over time (Ruben, 2005). While not consciously designed to do so, Mount Royal followed many of the guidelines and lessons shared by both Torraco and Hoover (2005) and Ruben (2005). They engaged external experts to assist in a needs assessment however they did not use an externally prescribed solution. They used the consultants' advice to inform an internal 
representative diagnostic team who were mandated to complete a needs assessment and make context sensitive recommendations. The V.P. Academic, who structurally sits two positions above Department Heads, fully supported the initiative. As evidenced in the case via the team processes, the leadership of the task groups appears to have created a safe and focused environment that balanced the need for task completion and continuous learning. Continuous communication between various interest groups - across, up and down the institutional structure ensured the V.P. Academic and task teams were constantly monitoring tensions, gathering input and adjusting plans. An awareness of the required balance between a centralized and decentralized approach was evidenced by the implementation taskforce supporting self selection on most implementation taskforce issues, but then selecting members for more complex or sensitive issues such as the creation of a performance planning and evaluation process for Department Heads. The persistence and motivation of the team has certainly been evidenced by both the number of changes implemented and the time the changes have taken.

Many of conditions described by Hackman and Edmondson's (2008) also appear to have been in place. The chartering of four separate groups: a diagnostic taskforce, an implementation taskforce and two ongoing sustaining groups with specific purposes gave each group clear boundaries and compelling and meaningful tasks which certainly required significant effort and used the members' knowledge, skill, and experience to complete. Oversight and steering opportunities were in place while, for the most part, the groups were given autonomy and they were left alone to do their work. However, although the desired Department Head role changes were created by a group of Heads themselves and OD guidelines, conditions and lessons learned 
were followed; implementing changes, has been a slow and complicated process, one that has required extensive consultation and communication.

In many ways this change initiative for the Department Head role has run parallel to the institution's transition from a college to a university. Initiatives, plans and goals are created, but they evolve over time and are not always implemented as originally envisioned. In the broader case of legislated institutional mandate shifts, official paper work may change an institutions status or label overnight, however it is the evolution and changes implemented by the institutional membership over time that appear to create an institutions unique character.

\section{Implications for organization development in higher education}

\section{Balancing steering and autonomy through group structures}

The structure of the various task groups and committees, the creation and disbandment of groups over time and a conscious effort to carry over a portion of the members from the various groups enabled Mount Royal University's change effort. These structures also provided a less invasive way for senior leadership to monitor and steer the work while still providing autonomy to complete the tasks. The findings suggest that group structures can play a valuable role in implementing OD initiatives. For example, creating a diagnostic team at the onset of an OD initiative requires a limited commitment of time and resources and it provides a logical check point to review the direction of the group. In addition diagnostic group membership requires a limited time commitment. This allows both the group member and the group leadership to determine if ongoing participation in the larger scope implementation group would be a good fit. 
Moreover, using the vetted and approved recommendations of a diagnostic group as the starting point for an implementation group creates a very natural link and builds on the work of the previous group (Ruben, 2005).

\section{Engage and enable the change targets as change agents}

At Mount Royal, Department Heads themselves have been the primary architects and implementers of the changes to their role. Other key staff members and administrators have been full members of the groups but as a minority of total membership. This suggests that the tensions inherent in leading universities centers on balancing the demands between administrative control and faculty autonomy (Bennett, 1998; Birnbaum, 1998; Birnbaum, 1992; Brown and Moshavi, 2002, cited by Blackmore, 2007, p. 400). It has been further suggested that, "pressure to participate needs to come from peers as well as from the top" (Turnbull and Edwards, 2005, p. 409). By engaging and enabling change targets as change agents, both of these suggestions may be addressed. While not all change initiatives require or have the benefit of allowing for the amount of time and effort outlined in the Mount Royal case, we further suggest that organizing groups of change targets as change agents is in alignment with academic traditions and values. Therefore it may be a powerful change approach for academic institutions working towards large scale shifts in culture and behaviour.

\section{Call for teamwork in higher education}


"When tasks are complex, large in scale or scope, or require inputs from multiple sources; groups are likely to be an effective way to get tasks accomplished" (Hackman and Edmondson, 2008, p.168). The university context certainly has been shown to be one that is riddled with complexity (Blackmore, 2007) and many of the challenges facing higher education institutions today are large in scale and require input from multiple sources to find sustainable solutions.

Teamwork in complex organizations is challenging. Institutions may benefit by broadly increasing member knowledge, competencies and experience with team work, team dynamics and an understanding of how groups can successfully be used as agents of change. There is a significant knowledge base to be leveraged which could further enable academic leaders, faculty and staff to act as agents of change. This knowledge, skills and abilities would provide critical capabilities that may help enable institutions to address many of the changes and challenges ahead.

\section{Conclusion}

This article provides a detailed description and analysis of how groups were used as change agents for an organization development initiative in a higher education context. More specifically it describes how one institution, Mount Royal University, used groups of Head's as change agents to address the key tensions associated with the Department Head role. 
Mount Royal first created a group to identify and diagnose the most consequential issues for their Department Heads. Next they created an intervention group to implement the recommendations identified by the diagnostic group. They further created two additional groups, ongoing chartered committees, to sustain the change.

The structure of these groups (a diagnostic group, then an implementation group, followed by ongoing maintenance groups) has enhanced leadership oversight and steering in a less direct or less invasive manner. Enabling and engaging the targets of change as the agents of change as demonstrated in the Mount Royal University case appears to have reduced change resistance and increased the likelihood of sustainable change. The findings also suggest that developing institution wide competencies associated with team work, team dynamics and how groups can be successfully used as agents of change may enable institutions to address many of the changes and challenges ahead.

\section{References}

Baldridge, J.V. 1971. Power and conflict in the university: Research in the sociology of complex organizations. New York: Wiley \& Sons.

Barker, B. 2006. Rethinking leadership and change: a case study in leadership succession and its impact on school transformation. Cambridge Journal of Education, 36, 17.

Beckhard, R. 1969. Organization development: Strategies and models, Reading, Addison-Wesley.

Beer, M. 1980. Organization change and development: A systems view, Glenview, Scott, Foresman.

Beer, M. \& Nohria, N. (eds.) 2000. Breaking the Code of Change, Boston: Harvard Business School Press.

Berdrow, I. 2010. King among Kings: Understanding the Role and Responsibilities of the Department Chair in Higher Education. Educational Management Administration \& Leadership, 38, 16.

Blackmore, P. 2007. Disciplinary difference in academic leadership and management and its development: a significant factor? Research in post-compulsory education, 12, 15.

Bryman, A. 2007. Effective leadership in higher education: a literature review. Studies in Higher Education, 32, 18.

Clark, B.R. 1983. The higher education system: Academic organization in cross-national perspective. Berkely: University of California Press. 
Clark, B.R. 1989. Creating entrepreneurial universities: Organizational pathways of transformation. Oxford: Pergamon Press.

Chu, D. 2006. Leading and Managing Academic Departments, Bolton, Anker Publishing Company.

Cohen, A. R., Fetters, M. \& Fleischmann, F. 2005. Major change at Babson College: curricular and administrative, planned and otherwise. Advances in Developing Human Resources, 7, 14.

Cummings, T. G. (ed.) 2008. Handbook of Organization Development, Thousand Oaks: Sage Publications, Inc.

Floyd, W.W \& Wooldridge, B. 1996. The strategic middle manager: How to create and sustain competitive advantage. San Fransisco: Jossey-Bass.

Hackman, J. R. \& Edmondson, A. C. 2008. Groups as Agents of Change. In: CUMMINGS, T. G. (ed.) Handbook of Organization Development. Thousand Oaks: Sage Publications, Inc.

Henkel, M. 2000. Academic identities and policy change in higher education, London, Jessica Kingsley.

Huisman, J., Norgard, J., Gulddahl-Rasmussen, J. \& Stensaker, B. 2002. Alternative universities revisited: A study of the distinctiveness of universities established in the spirit of 1968. Tertiary Education and Management, 8 (3), 316-332.

Kezar, A., Bertram Gallant, T. \& Lester, J. 2011. Everyday people making a difference on college campuses: the tempered grassroots leadership tactics of faculty and staff. Studies in Higher Education, 36, 129-151.

Kezar, A. \& Eckel, P. 2002. The Effect of Institutional Culture on Change Strategies in Higher Education: Universal Principles or Culturally Responsive Concepts? The Journal of Higher Education, 73, 26.

Kotter, J. P. \& Cohen, D. S. 2002. The Heart of Change, Boston, Harvard Business School Press.

Lees, N. D. 2006. Chairing Academic Departments, Bolton, Anker Publishing Company, Inc.

London, C. L. 2011. Development of an evaluation framework for the head of department role. Quality in Higher Education, 17, 15.

Mclean, G. N. 2005. Doing organization development in complex systems: The case at a large U.S. research, land-grant university. Advances in Developing Human Resources, 7, 13.

Mcroy, I. \& Gibbs, P. 2009. Leading Change in Higher Education. Educational Management Administration \& Leadership.

Meek, V.L. Goedegebuure, L., Santiago, R. \& Carvalho, T. 2010 (Eds). The changing dynamics of higher education middle mangement. Dordrecht: Springer.

Ruben, B. 2005. The Center for Organizational Development and Leadership at Rutgers University: A Case Study. Advances in Developing Human Resources, 7, 23.

Seagren, A. T., Creswell, J. W. \& Wheeler, D. W. 1993. The Deartment Chair: New Roles, Responsibilities and Challenges. ASHE-ERIC Higher Education Report No.1. Washington. D.C: The George Washington University, School of Education and Human Development.

Spiller, D. 2010. Language \& academic leadership: exploring \& evaluating the narratives. Higher Education Research \& Development 19, 14.

Sporn, B. 1999. Adaptive university structures: An analysis of adaptation to socioeconomic environments of US and European universities. London: Jessica Kingsley.

Torraco, R., J. \& Hoover, R., E. 2005. Organization Development and Change in Universities: Implications for Research and Practice. Advances in Developing Human Resources, 7, 422-437.

Tucker, A. L., Nembhard, I. M. \& Edmondson, A. C. 2007. Implementing new practices: An empirical study of organizational learning in hospital intensive care units. Management Science, 53, 14.

Turnbull, S. \& Edwards, G. 2005. Leadership Development for Organizational Change in a New U.K. University. Advances in Developing Human Resources, 7, 396-413. 